OPEN ACCESS

Edited by:

Christoph Mueller,

University of Bern, Switzerland

Reviewed by:

Gerhard Rogler,

University of Zurich, Switzerland

Tanima Bose,

Ludwig Maximilian University of

Munich, Germany

*Correspondence:

Joseph A. Murray

Murray.Joseph@mayo.edu

Specialty section: This article was submitted to

Mucosal Immunity,

a section of the journal

Frontiers in Immunology

Received: 16 June 2020 Accepted: 05 October 2020 Published: 28 October 2020

Citation:

Marietta E, Mangalam AK, Taneja V and Murray JA (2020) Intestinal

Dysbiosis in, and Enteral Bacterial Therapies for, Systemic Autoimmune Diseases.

Front. Immunol. 11:573079. doi: 10.3389/fimmu.2020.573079

\section{Intestinal Dysbiosis in, and Enteral Bacterial Therapies for, Systemic Autoimmune Diseases}

\author{
Eric Marietta ${ }^{1,2,3}$, Ashutosh K. Mangalam ${ }^{4}$, Veena Taneja ${ }^{2}$ and Joseph A. Murray ${ }^{1,2 *}$ \\ 1 Department of Gastroenterology and Hepatology, Mayo Clinic Rochester, Rochester, MN, United States, ${ }^{2}$ Department of \\ Immunology, Mayo Clinic Rochester, Rochester, MN, United States, ${ }^{3}$ Department of Dermatology, Mayo Clinic Rochester, \\ Rochester, MN, United States, ${ }^{4}$ Department of Pathology, University of lowa, lowa City, IA, United States
}

Recent studies have shown that a number of common autoimmune diseases have perturbations of their intestinal microbiome (dysbiosis). These include: Celiac Disease (CeD), Multiple Sclerosis (MS), Rheumatoid Arthritis (RA), Sjogren's Syndrome (SS), and Type 1 diabetes (T1D). All of these have intestinal microbiomes that are different from healthy controls. There have been numerous studies using animal models of single probiotics (monoclonal) or mixtures of probiotics (polyclonal) and even complete microbiota transfer (fecal microbial transfer-FMT) to inhibit or delay the onset of autoimmune diseases such as the aforementioned common ones. However, proportionally, fewer clinical trials have utilized monoclonal therapies or FMT than polyclonal therapies for treating autoimmune diseases, even though bacterial monotherapies do inhibit the development of autoimmune diseases and/or delay the onset of autoimmune diseases in rodent models of those autoimmune diseases. In this review then, we review the previously completed and currently ongoing clinical trials that are testing bacterial therapies (FMT, monoclonal, and polyclonal) to treat common autoimmune dseases and discuss the successes in using bacterial monotherapies to treat rodent models of these common autoimmune diseases.

Keywords: bacterial, monotherapies, autoimmune, microbiome, probiotic, treatment

\section{INTRODUCTION}

The definition of autoimmune disease first arose with Dr. Paul Ehrlich, wherein he described the condition as "horror intoxicus" (1). Currently Medline Plus.gov has the definintion of autoimmunity as "when the body's immune system attacks and destroys healthy body tissue by mistake" (https://medlineplus.gov/ency/article/000816.htm), and the website for the American Autoimmune Related Diseases Association (AARDA) has over 100 diseases listed as being autoimmune (https://www.aarda.org/diseaselist/). Many studies have been done where probiotics have been given to animal models of the less common autoimmune diseases. The most common autoimmune diseases as listed by Medline Plus.gov are: Addison disease, Celiac Disease, dermatomyositis, Graves disease, Hashimoto thyroiditis, multiple sclerosis, Myasthenia gravis, Pernicious anemia, Reactive arthritis, Rheumatoid arthritis, Sjogren syndrome, Systemic lupus erythematosus, and type 1 diabetes. This review will focus on these common autoimmune diseases, 
and more specifically, those autoimmune diseases that have had clinical trials conducted (or are being conducted) to treat autoimmune patients with bacterial therapies (fecal microbial transplantation and probiotic) as listed by clinicaltrials.gov. This therefore excludes Addison disease, dermatomyositis, Graves disease, Hashimoto Thyroiditis, Myasthenia gravis, Pernicious anemia, and Systemic Lupus Erythematosus.

The composition of the intestinal microbiome of many autoimmune diseases, including celiac disease (CeD), multiple sclerosis (MS), rheumatoid arthritis (RA), Sjogren's syndrome (SS), and type 1 diabetes (T1D) has been characterized predominantly using $16 \mathrm{~s}$ rDNA sequencing of stool samples. Studies have demonstrated that alterations of the fecal intestinal (colonic) microbiome (dysbiosis) exist in patients that have $\mathrm{CeD}$, MS, RA, SS, and/or T1D, and that MS patients may have a uniform decrease in Prevotella (2-14).

One study done with T1D patients demonstrated that there was also dysbiosis in the small bowel of T1D patients as compared to controls (15). Additionally, a study with duodenal biospises of MS patients found dysbiosis in their small intestine as well (16). In addition, a number of studies found dysbiosis in the small bowel of CeD patients $(6,7,17-21)$. All of these studies clearly demonstrate that patients with these autoimmune diseases have dysbiosis in their colonic microbiomes, where the composition of their fecal microbiome is different from controls such as first degree relatives or healthy controls. The data for dysbiosis in the small intestine of $\mathrm{CeD}$ is strong, but more research needs to be done with MS, RA, SS, and T1D.

It has been often assumed microbial therapies work by normalizing the resident microbiota, and hence, prevent or treat autoimmune diseases that have associated dysbiosis. How the change in the composition of the intestinal microbiome due to microbial therapy would exert changes in the systemic immune system has been a focus of many rodent model studies. At least two pathways have been identified. The first is through Pattern Recognition Receptors (PRRs), such as Toll-Like Receptors (TLRs), on different cell types that interact with bacteria in the lumen, and the PRRs would detect and bind to Microbe Associated Molecular Patterns (MAMPs) expressed by the bacteria in the intestine $(22,23)$. Rodent models for MS have demonstrated a role for TLR2 in controlling and treating disease $(24,25)$.

The second pathway would be through the production of Short Chain Fatty Acids (SCFAs) by the bacteria. The SCFAs would bind to SCFA receptors expressed by the responsive host immune cell, resulting in a phenotypic change to being either regulatory or inflammatory, and deficits in SCFA production have been identified in multiple sclerosis $(5,26,27)$.

However, normality for the human intestinal microbiome is unclear and varies greatly by geography, diet, and other external factors (28-31). Even age affects the composition of the human intestinal microbiome, such that there are at least four distinct age groups in which the human intestinal microbiome is different (infant, pre-adolescent, adult, elderly) (32-35). Genetics play a crucial role as well, and rodent models have demonstrated that even the smallest alterations in the genetic background can lead to changes in the composition of the intestinal microbiome (3639). Differences in the composition of the intestinal microbiome that are due to the effect of age, diet, geography, and host genetics, could potentially also contribute to different responses to different bacterial therapies, although the specific differences as a consequence of these factors, especially age and genetics, in response to bacterial therapies have not been rigorously addressed, especially in humans.

Despite the complexity of the effects of probiotic treatment, the concept of probiotics as being beneficial in helping the intestine stabilize bacterial content (reach homeostasis) is currently well known publically. The concept of how much bacteria and which types of bacteria are needed to achieve homeostasis is not as well known publically, nor determined in a truly rigorous scientific manner (Figure 1). The transfer of complete microbial content, or Fecal Microbial Transplantation (FMT), interestingly was done as long ago as the fourth century in China to treat diarrhea, constipation, and abdominal pain (40). In this type of treatment today, all of the fecal bacteria is transferred. Currently, the greatest success story of FMT is with the treatment of patients infected with Clostridium difficile ( $C$. difficile) $(41,42)$. Mixes of probiotics, such as in the form of yogurt, are not a complete mix of the bacteria found in the digestive tract of humans. And at the opposite spectrum, there are many studies done with rodents where only one bacteria is provided to the animal. With autoimmune diseases, FMTs and probiotics (both mixes and single strains) are being used in clinical trials for treating patients with autoimmune disease. In addition, there is a fourth category, that of bioengineered probiotics/bacteria that secrete proteins to reduce autoimmune responses. This will be highlighted in the last section on type 1 diabetes, as there is currently an ongoing clinical trial testing such a product. In order therefore to obtain insight into the effectiveness of the three main types of intestinal microbial treatments (complete, restricted mix, and monoclonal) used in treating autoimmune diseases, this review will systematically progress through each of the common autoimmune diseases that have had clinical trials conducted where probiotics (including FMTs) were given to autoimmune patients to treat their disease (CeD, MS, RA, SS, and T1D).

\section{AUTOIMMUNE DISEASES}

\section{Celiac Disease}

There is currently one clinical trial (NCT 04014413) that is recruiting Celiac patients for conducting FMT. This study is also determining the efficacy of FMT for a number of other autoimmune and inflammatory disorders and is still recruiting patients. No data has been released yet. In contrast, there have been many randomized, double blind, placebo clinical trials testing monoclonal and polyclonal bacterial therapies with Celiac Disease that have been completed and results published, with at least four (43-46) that are monoclonal and three polyclonal (47-49), with the earliest results published as far back as 2013 (46). Bacteria used in the bacterial monotherapies 


\section{How many probiotic bacterial species/lines does it take to induce systemic immune responses that will suppress autoimmunity in affected organs?}

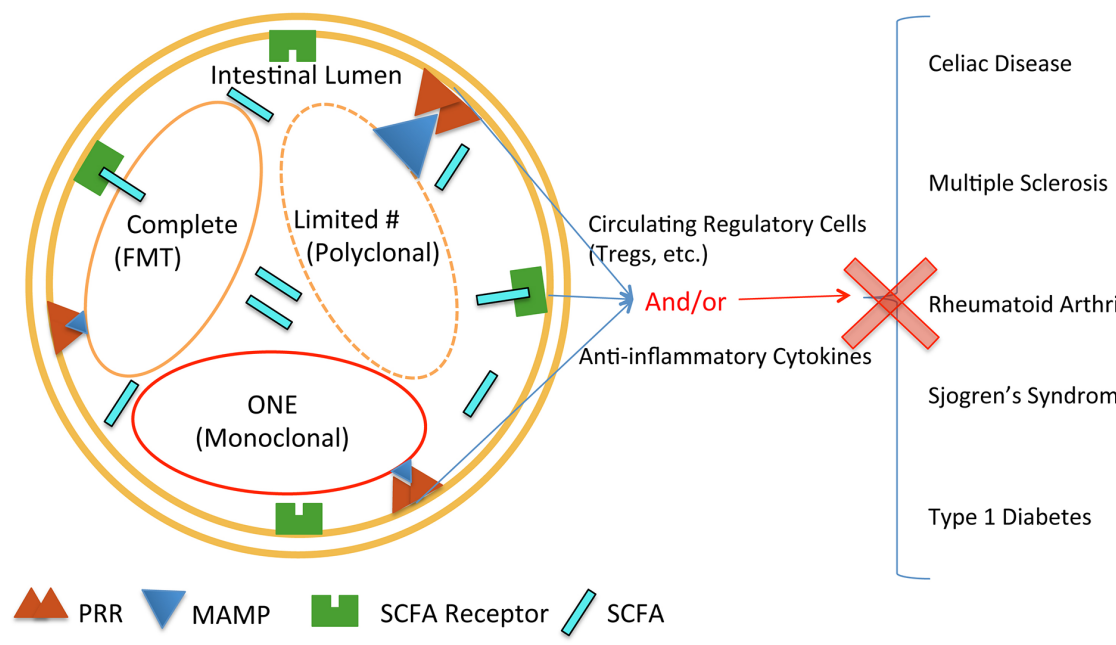

FIGURE 1 | Mechanisms by which probiotic bacterial therapies can affect inflammation at intestinal and extraintestinal sites. Depicted in the intestinal lumen are the three main categories of bacterial groups currently used in clinical trials, reflecting quantity: complete, restricted mix (Limited\#/Polyclonal), and one (monoclonal). PRR, pattern recognition receptor; MAMP, microbe associated molecular pattern; SCFA, short chain fatty acid; SCFAR, SCFA Receptor.

were Bifidobacterium infantis $(45,46)$, Bifidobacterium breve (43), and Bifidobacterium longum (44). The rest were mixes of two or more bacterial strains (47-49). In the clinical trial with $B$. breve (43), circulating levels of TNF $\alpha$ present in children on a GFD were decreased with the administration of $B$. breve. This clinical trial was based in part on the following mouse models $(50,51)$, wherein $B$. breve induced regulatory T cells (Tregs) in mice. With the administration of $B$. longum to children with CeD (44) there was a significant reduction in the number of circulating $\mathrm{CD} 3+\mathrm{T}$ lymphocytes and a slight reduction in the level of TNF $\alpha$. This was based in large part on a previous study with a rat model of enteropathy, wherein administration of $B$. longum to the rats increased IL10 and decreased TNF $\alpha$ (52). With the administration of $B$. infantis to adults (46), there was an improvement in the gastrointestinal rating scale for indigestion, constipation, and reflux, and a later publication (45) found that $B$. longum affected the innate immune system of patients by decreasing $\alpha$-defensin 5. Previous studies had shown that the administration of $B$. infantis to mouse models of colitis and bacterial inflammation (53-55) had increased the number of Tregs and downregulated inflammatory cytokines. In the publication on a polyclonal treatment by Francavilla et al. (47), five strains of lactic acid bacteria and bifidobacteria were given to adult Celiac patients with IBS like symptoms. The administration of the probiotic mix led to a significant decrease in the gastrointestinal symptom rating scale, whereas the placebo did not. In the publication on the administration of two lactobacillus strains (L. plantarum HEAL9 and $L$. paracasei 8700:2) to children who were tTG IgA positive but still on a gluten containing diet, a detailed analysis of circulating lymphocytes was done by flow cytometry (48). The most notable change was a significantly reduced expansion of CD4+CD25 $+\mathrm{CD} 45 \mathrm{RO}+\mathrm{T}$ cells (effector and memory T cells) in the probiotic group (48). No significant change was observed in the levels of circulating anti tTG IgA levels (48). With the third clinical trial that administered a mix of probiotic bacteria, VSL\#3 was administered to adult $\mathrm{CeD}$ patients who still had gastrointestinal symptoms, despite being on a gluten free diet (49). CeD patients who received the VSL\#3 probiotic mix had no improvement in clinical gastrointestinal symptoms over the placebo group (49). The CeD patients also did not have significant changes to their gut microbiome as measured by microscopic and molecular analysis (49).

\section{Multiple Sclerosis}

Three clinical trials have been set up for conducting FMT with MS patients (NCT03183869), (NCT03975413), and (NCT04150549). Only 10 patients were recruited for the NCT03183869 study due to early termination (the primary investigator passed away), and no significant changes in circulating cytokines were observed (either inflammatory or anti-inflammatory) in the small number of patients. NCT04150549 has not started recruiting patients, and NCT03975413 is still active. Rodent models of FMT to treat EAE provide mixed results. One study with rats show that transfer of fecal microbiota of EAE resistant rats (Albino Oxford) to EAE rats ameliorates the disease in disease prone rats (Dark Agouti) (56). In contrast, another study observed that the transfer of fecal microbota from naïve mice to EAE mice did not ameliorate disease (37). It was only when fecal microbiota was transferred from CD44 knock out mice that disease was ameliorated (37). 
No clinical trials with bacterial monotherapies have been conducted for MS patients. However, in one randomized, double blind, placebo-controlled study conducted at the Islamic Azad Medical Center, capsules containing probiotic bacteria, Lactobacillus acidophilus, Lactobacillus casei, Bifidobacterium bifidum and Lactobacillus fermentum, were administered to relapsing remitting MS patients (RRMS) (57). Patients who received the probiotic mix had significantly decreased EDSS (expanded disability status scale) and DASS (depression, anxiety, and stress scale). Another study conducted at Harvard Medical Center administered a very common and commercially available probiotic mix, called Visbiome, which was previously called VSL\#3, to MS patients and healthy controls (58). Since no placebo was tested, this study was not a randomized double blind placebo trial. The mix contained 8 different strains of bacteria: Lactobacillus paracasei DSM 24734 , Lactobacillus plantarum DSM 24730, Lactobacillus acidophilus DSM 24735, and Lactobacillus delbruckeii subspecies bulgaricus DSM 24734), Bifidobacterium longum DSM 24736, Bifidobacteriuminfantis DSM 24737, and Bifidobacterium breve DSM24732), and Streptococcus thermophilus DSM 24731). RRMS patients who received Visbiome had changes to their gut microbiome composition, and healthy controls who received Visbiome had a decreased alpha diversity.

\section{Rheumatoid Arthritis}

One clinical trial (NCT03944096) is currently being conducted with testing FMT for treating RA patients. Its title is Efficacy and Safety of Fecal Microbiota Transplantation in Patients With Rheumatoid Arthritis Refractory to Methotrexate (FARM). No results have been posted yet though.

Only one group, at the Tehran University of Medical Sciences, has published results on providing a bacterial monotherapy for treating RA. They did a randomized, double- blind, placebocontrolled clinical trial using capsules that contained active $L$. casei (59). They obtained similar results with the women with $\mathrm{RA}$, in that inflammatory cytokines went down and antiinflammatory IL-10 went up. The disease scores for tender and swollen joints for the patients that received the L. casei were also decreased as compared to the placebo. L. casei was tested previously for its effectiveness in mouse and rat models of arthritis to decrease the inflammation of arthritis in those models (60-62). These studies showed that in the animal models, the administration of $L$. casei resulted in decreased incidence and development of arthritis that was associated with decreased production of inflammatory cytokines such as IFN $\gamma$, IL-17, and TNF $\alpha$, and a decrease in the production of anti collagen antibodies. A different randomized, double- blind, placebo-controlled clinical trial conducted at the Kashan University of Medical Sciences, RA patients were given L. casei in addition to Lactobacillus acidophilus and Bifidobacterium bifidum in a capsule. The disease activity score of 28 joints was decreased significantly in the probiotic treated group; however, the tender and swollen joints scores individually were not decreased (63). A second group gave Lactobacillus rhamnosus GR-1 and Lactobacillus reuteri RC-14 in capsules to RA patients in a randomized, double- blind, placebo-controlled clinical trial conducted at the St. Joseph's Health Care in London, Ontario, Canada (64). They did not observe an overall clinical improvement in the probiotic arm, but they did find that blood levels of GMCSF, MIP1 $\alpha$, TNF $\alpha$, IL-6, IL12p70, IL-15, and IL-17, as determined by multiplex immunoassay, were decreased in the probiotic arm.

Thus, although there is only one published clinical trial for administering a bacterial monotherapy to RA patients, the results do suggest that such therapies can be effective in reducing inflammation in RA patients and that this is associated with increased systemic production in anti inflammatory cytokines such as IL10 and decreased production of inflammatory cytokines.

\section{Sjogren's Syndrome}

One clinical trial for treating Sjogren's Syndrome with FMT has been completed (NCT03926286). No results have been posted yet though. Results in rodent models of fecal microbiota transfer to treat SS has been promising though. In one study, the transfer of fecal microbiota of disease-free mice to a mouse model of SS did ameliorate the disease (65). A second study using a different mouse model of SS had similar results in that transfer of fecal microbiota from disease free mice reverted the disease along with a decrease in the pathogenic CD4+IFN $\gamma+\mathrm{T}$ cells (66).

No clinical trials for SS using a monoclonal bacterial therapy have been established yet. However, there is a clinical trial set up to determine the efficacy of treating oral candidiasis in SS (NCT03840538) with probiotics, and this has been completed and results published (67). Here a mix of probiotics was given (Lactobacillus acidophilus, Lactobacillus bulgaricus, Streptococcus thermophilus and Bifidobacterium bifidum) to SS patients to determine if that would decrease candidial load. Results from the study showed that this mix did provide a statistically significant decrease in the candidial load from baseline to treatment end, and the difference was not statistically significant in the placebo group. There is one publication that showed that administering a probiotic mix to a mouse model of dry eye, the main symptom of primary SS, does have a beneficial effect (68). In this study, a mix called IRT5 was used (Bifidobacterium bifidum, Lactobacillus acidophilus, Lactobacillus casei, Lactobacillus reuteri, and Streptococcus thermophilus) (68). The administration of these bacteria orally by gavage led to increased levels of CD11c+ and CD11b+ cells in the spleen and increased levels of IL-10 along with decreased levels of IL1 $\beta$ in the conjuctiva and cornea of the eye (68).

\section{Type I Diabetes}

Currently there is one clinical trial designed to determine the benefit of FMT for treating T1D (NCT04124211). This is still recruiting patients, so no results have been posted.

With T1D, only one bacterial monotherapy placebocontrolled, double blind, randomized clinical trial has been completed and results published (69). In that study, conducted at the University Medical Center in Ahvaz, Iran, patients were administered a synbiotic mixture of Lactobacillus sporogenes and a corresponding prebiotic, fructooligosaccharide for 8 weeks to children diagnosed with T1D (69). The synbiotic mix improved the following glycemic indices in the children as compared to a placebo control group: FBG, HbA1c, insulin, hs-CRP, and TAC. 
This was preceded by a study in an animal model in which Bifidobactera bifidum and Lactobacillus sporogenes were administered separately to rats that developed paw edema due to injection with carrageenan (70). Administration of either bacterial strain alone to the rats resulted in decreased paw thickness, and increased physical activity as determined by a stair climbing assay and motility assay (70). Another clinical trial is set up to administer a bacterial monotherapy (NCT03961347), but it is still recruiting patients at the University of Florida. In this clinical trial, L. johnsonii is being administered to adults with T1D. This clinical trial was preceded by studies in animal models as well (71-76). Three other clinical trials have been set up to test the effect of polymicrobial therapies on T1D, but no publications on the results have yet emerged (NCT03032354 (77), NCT03880760, and NCT03423589). The first multiple bacterial therapy clinical trial (NCT03032354) was set up by Groele et al. to be conducted at the Medical University of Warsaw, Poland and the Department of Endocrinology and Diabetology, Children's Memorial Health Institute in Warsaw, Poland (77). Children with T1D were to be recruited and given a mix of L. rhamnosus and $B$. lactis Bb12. The second one with multiple bacteria (NCT03880760), was set up to be conducted at the China Medical University Hospital in Taichung, Taiwan. It was designed to treat T1D children with a mix of Lactobacillus salivarius + Lactobacillus johnsonii + Bifidobacterium lactis. The third multiple bacterial therapy clinical trial, NCT03423589, was designed to administer to T1D patients VSL\#3 (Visbiome) at the Medical School of Wisconsin. As described previously, VSL\#3 is a mix of 8 bacterial strains. Overall then, only one clinical trial has been completed and published on the efficacy of administering bacteria to treat T1D, and this used a bacterial monotherapy and had promising data.

NCT03751007 is a clinical trial that is utilizing bacteria from the fourth category, genetically engineered bacterial, Lactococcus lactis that secretes proinsulin and IL10 (AG019-Precigen Actobio T1D, LLC), and is currently (as of this publication) being conducted at 18 sites in the United States and Belgium. This genetically modified bacterial strain was tested first in NOD mice (76). Administration of the L. lactis secreting proinsulin and IL10 along with anti CD3, substantially decreased hyperglycemia in the NOD mice (76).

\section{CONCLUSIONS/DISCUSSION}

Of the five common autoimmune diseases that had clinical trials to test bacterial therapies (CeD, MS, RA, SS, and T1D), all five had FMT being tested. One for Celiac disease, three for MS, one for RA, one for SS, and one for T1D (7 in total). Only one had truly completed (NCT03926286), which is the one determining the efficacy of treating SS with FMT. No results from that clinical trial have been posted yet. Another one (NCT03183869) for MS had terminated early, but had posted results on circulating cytokines. Thus, it is too early to make any predictions on the efficacy of treating autoimmune diseases with FMT; however, there is a lot of effort going into answering this question.
There were 3 clinical trials that use a restricted number of probiotic bacteria for Celiac Disease, 2 for MS, 3 for RA, 1 for SS, and 3 for T1D (12 in total). This is about twice as many as the number of clinical trials to evaluate the efficacy of treating autoimmune diseases with FMT. These gave mixed results in that some studies showed improvement in gastrointestinal symptoms, but others did not. Also too, not all of the studies evaluated immune responses in a similar way, making it difficult to determine if there was a common response by the immune system to these mixes of probiotic bacteria, most especially in those trials where no beneficial changes were observed to occur in gastrointestinal symptoms.

For the administration of monoclonal therapies to patients with autoimmune diseases, there were 4 with celiac disease, 0 for MS, 1 for RA, 0 for SS, and 2 for T1D (7 total). Results from the Celiac monoclonal clinical trials had results that showed that administrating monoclonal therapies decreased levels of sera TNF $\alpha$ and circulating CD3 + T cells. Also too $\alpha$-defensin 5 of the innate immune system was decreased. In addition, gastrointestinal symptoms improved with the administration of monoclonal bacterial therapy in $\mathrm{CeD}$ patients. Similarly in $\mathrm{RA}$ patients, using only L. casei, inflammatory cytokines went down and anti-inflammatory IL10 went up. Symptoms and disease scores improved as well. With the one monoclonal therapy on T1D that was published, glycemic indices were improved with the administration of $L$ sporogenes. Overall then, there is a deficit in the data on the outcomes of monoclonal bacterial therapies for treating autoimmune patients, but the limited data suggests that, at least with $\mathrm{CeD}$ and $\mathrm{RA}$, there is a decrease in systemic inflammatory cytokines that is associated with an increase in anti inflammatory cytokines. The rodent models of these diseases and the administration of these same monoclonal bacteria support these findings as well as suggest that regulatory $\mathrm{T}$ cells are increased by the administration of these bacteria. It should be noted though, that the rodent models of autoimmune diseases treated with the probiotic mixes had similar results as well.

In addition, there are many other rodent models of monoclonal therapies to treat these common autoimmune diseases, all of which have similar findings in that regulatory $\mathrm{T}$ cells are increased along with anti inflammatory cytokines and other regulatory cells. One example is with Prevotella histicola in a CIA model, in which $P$. histicola induced IL-10, an antiinflammatory cytokine, in the intestines (jejunum) of the treated mice (78). Serum levels of IL10 also increased after two weeks of administering $P$. histicola, as well as levels of regulatory $\mathrm{T}$ cells and a corresponding decrease in levels of Th17 cells (78). With the EAE model, $P$. histicola induced the production of IL10 in dendritic cells and macrophages and increased levels of regulatory $\mathrm{T}$ cells (79). Both of these studies demonstrate that $P$. histicola has anti-inflammatory properties that lead to the generation of regulatory $\mathrm{T}$ cells. With $\mathrm{T} 1 \mathrm{D}$, there have been a couple of studies that have shown that bacterial monotherapies work to decrease the incidence of T1D in NOD mice by increasing the levels of regulatory $\mathrm{T}$ cells $(80,81)$. In the one study with Clostridium, the Tregs would be generated in the intestine and then migrate to the pancreatic lymph nodes (80). 
And with the study with Akkermansia muciniphilia, increased numbers of Tregs generated by A. muciniphilia were associated with increased levels of IL10 and TGF $\beta$ (81). Other rodent studies that have monoclonal therapies for Celiac Disease include treatment with P. histicola (82), B. longum (52, 83, 84), L. rhamnosus (85) and L. casei (86) For MS, there are rodent EAE models using Lactobacillus reuteri (87) and Lactobacillus plantarum (88). For RA, there are rodent models using L. casei (60, 62, 89), Bifidobacterium animalis (90), Lactobacillus fermentum (91), L. plantarum (92), L. rhamnosus (93, 94). For T1D, there is another rodent model that uses a monoclonal therapy, but a different species of Lactobacillus, Lactobacillus brevis (95). Thus, there is a large number of probiotic monotherapies for autoimmune diseases that have been tested in rodent models, but relatively few have been incorporated into clinical trials.

As to the fourth category of probiotics, that of bioengineered bacteria that secrete specific disease associated antigens in order to suppress disease activity, there are three rodent models. There is the previously mentioned study for T1D (76) that used Lactococcus lactis that secreted proinsulin and IL10. There is also one for a mouse model of $\mathrm{CeD}$ in which Lactococcus lactis that secreted a gliadin epitope was used (96). The third is a study that generated lactobaccili to express EAE antigens and then administered that to a rat EAE model (97). All three of these models using recombinant bacteria showed a decrease in disease activity with the administration of the recombinant bacteria to the rodent.

In summary, there are almost twice as many clinical trials that are being, or have been conducted, that use probiotic mixes as

\section{REFERENCES}

1. Living with the enemy. Nat Immunol (2018) 19:658-8. doi: 10.1038/s41590018-0153-8

2. Chen J, Wright K, Davis JM, Jeraldo P, Marietta EV, Murray J, et al. An expansion of rare lineage intestinal microbes characterizes rheumatoid arthritis. Genome Med (2016) 8:43. doi: 10.1186/s13073-016-0299-7

3. Sun Y, Chen Q, Lin P, Xu R, He D, Ji W, et al. Characteristics of Gut Microbiota in Patients With Rheumatoid Arthritis in Shanghai, China. Front Cell Infect Microbiol (2019) 9:369. doi: 10.3389/fcimb.2019.00369

4. Chen J, Chia N, Kalari KR, Yao JZ, Novotna M, Soldan MM, et al. Multiple sclerosis patients have a distinct gut microbiota compared to healthy controls. Sci Rep (2016) 6:28484. doi: 10.1038/srep28484

5. Zeng Q, Gong JL, Liu XY, Chen C, Sun XB, Li HJ, et al. Gut dysbiosis and lack of short chain fatty acids in a Chinese cohort of patients with multiple sclerosis. Neurochem Int (2019) 129:104468. doi: 10.1016/j.neuint.2019. 104468

6. Bodkhe R, Shetty SA, Dhotre DP, Verma AK, Bhatia K, Mishra A, et al. Comparison of Small Gut and Whole Gut Microbiota of First-Degree Relatives With Adult Celiac Disease Patients and Controls. Front Microbiol (2019) 10:164. doi: 10.3389/fmicb.2019.00164

7. Garcia-Mazcorro JF, Rivera-Gutierrez X, Cobos-Quevedo OJ, Grube-Pagola P, Meixueiro-Daza A, Hernandez-Flores K, et al. First Insights into the Gut Microbiota of Mexican Patients with Celiac Disease and Non-Celiac Gluten Sensitivity. Nutrients (2018) 10:1-19. doi: 10.3390/nu10111641

8. Higuchi BS, Rodrigues N, Gonzaga MI, Paiolo JCC, Stefanutto N, Omori WP, et al. Intestinal Dysbiosis in Autoimmune Diabetes Is Correlated With Poor there are that are using monoclonal bacterial therapies or FMTs. So far, there are few rodent models of FMTs to treat the common autoimmune diseases, and the results are mixed. FMT to treat SS in rodents has provided positive results, but FMT to treat EAE in rodents has provided contrasting results. In contrast, there is a large number of rodent models of autoimmunity that demonstrate that monoclonal bacterial therapies are effective in altering the systemic immune respone in autoimmunity. Many more clinical trials should be undertaken to conclusively determine the efficacy of monoclonal bacterial therapies in treating autoimmune diseases, especially with bacterial strains that have been tested in rodent models but not yet tested in clinical trials. Since FMTs are designed to transfer all of the microbial content to the recipient and there are a number of currently ongoing clinical trials, there should be enough data in the next five years to determine the efficacy of FMTs in treating autoimmunity.

\section{AUTHOR CONTRIBUTIONS}

All authors contributed to the article and approved the submitted version.

\section{FUNDING}

Funding is supported by grants from NIH (NIH- R01AI075262), Mayo Clinic, and the Department of Defense (DOD- W81XWH10-1-0257).

Glycemic Control and Increased Interleukin-6: A Pilot Study. Front Immunol (2018) 9:1689. doi: 10.3389/fimmu.2018.01689

9. Gursoy S, Kockar T, Atik SU, Onal Z, Onal H, Adal E. Autoimmunity and intestinal colonization by Candida albicans in patients with type 1 diabetes at the time of the diagnosis. Korean J Pediatr (2018) 61:217-20. doi: 10.3345/ kjp.2018.61.7.217

10. Huang Y, Li SC, Hu J, Ruan HB, Guo HM, Zhang HH, et al. Gut microbiota profiling in Han Chinese with type 1 diabetes. Diabetes Res Clin Pract (2018) 141:256-63. doi: 10.1016/j.diabres.2018.04.032

11. Mejia-Leon ME, Petrosino JF, Ajami NJ, Dominguez-Bello MG, de la Barca AM. Fecal microbiota imbalance in Mexican children with type 1 diabetes. Sci Rep (2014) 4:3814. doi: 10.1038/srep03814

12. Mendez R, Watane A, Farhangi M, Cavuoto KM, Leith T, Budree S, et al. Gut microbial dysbiosis in individuals with Sjogren's syndrome. Microb Cell Fact (2020) 19:90. doi: 10.1186/s12934-020-01348-7

13. Moon J, Choi SH, Yoon CH, Kim MK. Gut dysbiosis is prevailing in Sjogren's syndrome and is related to dry eye severity. PloS One (2020) 15:e0229029. doi: 10.1371/journal.pone.0229029

14. van der Meulen TA, Harmsen HJM, Vila AV, Kurilshikov A, Liefers SC, Zhernakova A, et al. Shared gut, but distinct oral microbiota composition in primary Sjogren's syndrome and systemic lupus erythematosus. J Autoimmun (2019) 97:77-87. doi: 10.1016/j.jaut.2018.10.009

15. Pellegrini S, Sordi V, Bolla AM, Saita D, Ferrarese R, Canducci F, et al. Duodenal Mucosa of Patients With Type 1 Diabetes Shows Distinctive Inflammatory Profile and Microbiota. J Clin Endocrinol Metab (2017) 102:1468-77. doi: 10.1210/jc.2016-3222

16. Cosorich I, Dalla-Costa G, Sorini C, Ferrarese R, Messina MJ, Dolpady J, et al. High frequency of intestinal TH17 cells correlates with microbiota alterations 
and disease activity in multiple sclerosis. Sci Adv (2017) 3:e1700492. doi: 10.1126/sciadv.1700492

17. Wacklin P, Kaukinen K, Tuovinen E, Collin P, Lindfors K, Partanen J, et al. The duodenal microbiota composition of adult celiac disease patients is associated with the clinical manifestation of the disease. Inflammation Bowel Dis (2013) 19:934-41. doi: 10.1097/MIB.0b013e31828029a9

18. Sanchez E, Donat E, Ribes-Koninckx C, Fernandez-Murga ML, Sanz Y. Duodenal-mucosal bacteria associated with celiac disease in children. Appl Environ Microbiol (2013) 79:5472-9. doi: 10.1128/AEM.00869-13

19. D’Argenio V, Casaburi G, Precone V, Pagliuca C, Colicchio R, Sarnataro D, et al. Metagenomics Reveals Dysbiosis and a Potentially Pathogenic N. flavescens Strain in Duodenum of Adult Celiac Patients. Am J Gastroenterol (2016) 111:879-90. doi: 10.1038/ajg.2016.95

20. Panelli S, Capelli E, Lupo GFD, Schiepatti A, Betti E, Sauta E, et al. Comparative Study of Salivary, Duodenal, and Fecal Microbiota Composition Across Adult Celiac Disease. J Clin Med (2020) 9:1-20. doi: 10.3390/jcm9041109

21. Schippa S, Iebba V, Barbato M, Di Nardo G, Totino V, Checchi MP, et al. A distinctive 'microbial signature' in celiac pediatric patients. BMC Microbiol (2010) 10:175. doi: 10.1186/1471-2180-10-175

22. Fischer M, Ehlers M. Toll-like receptors in autoimmunity. Ann N Y Acad Sci (2008) 1143:21-34. doi: 10.1196/annals.1443.012

23. Fawkner-Corbett D, Simmons A, Parikh K. Microbiome, pattern recognition receptor function in health and inflammation. Best Pract Res Clin Gastroenterol (2017) 31:683-91. doi: 10.1016/j.bpg.2017.11.001

24. Anstadt EJ, Fujiwara M, Wasko N, Nichols F, Clark RB. TLR Tolerance as a Treatment for Central Nervous System Autoimmunity. J Immunol (2016) 197:2110-8. doi: 10.4049/jimmunol.1600876

25. Wang Y, Telesford KM, Ochoa-Reparaz J, Haque-Begum S, Christy M, Kasper EJ, et al. An intestinal commensal symbiosis factor controls neuroinflammation via TLR2-mediated CD39 signalling. Nat Commun (2014) 5:4432. doi: 10.1038/ncomms5432

26. Mizuno M, Noto D, Kaga N, Chiba A, Miyake S. The dual role of short fatty acid chains in the pathogenesis of autoimmune disease models. PloS One (2017) 12:e0173032. doi: 10.1371/journal.pone.0173032

27. Marino E, Richards JL, McLeod KH, Stanley D, Yap YA, Knight J, et al. Gut microbial metabolites limit the frequency of autoimmune $\mathrm{T}$ cells and protect against type 1 diabetes. Nat Immunol (2017) 18:552-62. doi: 10.1038/ni.3713

28. Gaulke CA, Sharpton TJ. The influence of ethnicity and geography on human gut microbiome composition. Nat Med (2018) 24:1495-6. doi: 10.1038/ s41591-018-0210-8

29. Stearns JC, Zulyniak MA, de Souza RJ, Campbell NC, Fontes M, Shaikh M, et al. Ethnic and diet-related differences in the healthy infant microbiome. Genome Med (2017) 9:32:1-12. doi: 10.1186/s13073-017-0421-5

30. Niu J, Xu L, Qian Y, Sun Z, Yu D, Huang J, et al. Evolution of the Gut Microbiome in Early Childhood: A Cross-Sectional Study of Chinese Children. Front Microbiol (2020) 11:439. doi: 10.3389/fmicb.2020.00439

31. Jha AR, Davenport ER, Gautam Y, Bhandari D, Tandukar S, Ng KM, et al. Gut microbiome transition across a lifestyle gradient in Himalaya. PloS Biol (2018) 16:e2005396. doi: 10.1371/journal.pbio.2005396

32. Shenhav L, Furman O, Briscoe L, Thompson M, Silverman JD, Mizrahi I, et al. Modeling the temporal dynamics of the gut microbial community in adults and infants. PloS Comput Biol (2019) 15:e1006960. doi: 10.1371/ journal.pcbi.1006960

33. Radjabzadeh D, Boer CG, Beth SA, van der Wal P, Kiefte-De Jong JC, Jansen MAE, et al. Diversity, compositional and functional differences between gut microbiota of children and adults. Sci Rep (2020) 10:1040. doi: 10.1038/ s41598-020-57734-z

34. Hollister EB, Riehle K, Luna RA, Weidler EM, Rubio-Gonzales M, Mistretta $\mathrm{TA}$, et al. Structure and function of the healthy pre-adolescent pediatric gut microbiome. Microbiome (2015) 3:36. doi: 10.1186/s40168-015-0101-x

35. Claesson MJ, Cusack S, O'Sullivan O, Greene-Diniz R, de Weerd H, Flannery E, et al. Composition, variability, and temporal stability of the intestinal microbiota of the elderly. Proc Natl Acad Sci U.S.A. (2011) 108 Suppl 1:4586-91. doi: 10.1073/pnas.1000097107

36. Gomez A, Luckey D, Yeoman CJ, Marietta EV, Berg Miller ME, Murray JA, et al. Loss of sex and age driven differences in the gut microbiome characterize arthritis-susceptible ${ }^{\star} 0401$ mice but not arthritis-resistant ${ }^{\star} 0402$ mice. PloS One (2012) 7:e36095. doi: 10.1371/journal.pone.0036095
37. Chitrala KN, Guan H, Singh NP, Busbee B, Gandy A, Mehrpouya-Bahrami P, et al. CD44 deletion leading to attenuation of experimental autoimmune encephalomyelitis results from alterations in gut microbiome in mice. Eur J Immunol (2017) 47:1188-99. doi: 10.1002/eji.201646792

38. Moshkelgosha S, Masetti G, Berchner-Pfannschmidt U, Verhasselt HL, Horstmann M, Diaz-Cano S, et al. Gut Microbiome in BALB/c and C57BL/ 6J Mice Undergoing Experimental Thyroid Autoimmunity Associate with Differences in Immunological Responses and Thyroid Function. Horm Metab Res (2018) 50:932-41. doi: 10.1055/a-0653-3766

39. Korach-Rechtman H, Freilich S, Gerassy-Vainberg S, Buhnik-Rosenblau K, Danin-Poleg Y, Bar H, et al. Murine Genetic Background Has a Stronger Impact on the Composition of the Gut Microbiota than Maternal Inoculation or Exposure to Unlike Exogenous Microbiota. Appl Environ Microbiol (2019) 85:1-12. doi: 10.1128/AEM.00826-19

40. Wang JW, Kuo CH, Kuo FC, Wang YK, Hsu WH, Yu FJ, et al. Fecal microbiota transplantation: Review and update. J Formos Med Assoc (2019) 118 Suppl 1:S23-31. doi: 10.1016/j.jfma.2018.08.011

41. Kassam Z, Lee CH, Yuan Y, Hunt RH. Fecal microbiota transplantation for Clostridium difficile infection: systematic review and meta-analysis. Am J Gastroenterol (2013) 108:500-8. doi: 10.1038/ajg.2013.59

42. Allegretti JR, Mullish BH, Kelly C, Fischer M. The evolution of the use of faecal microbiota transplantation and emerging therapeutic indications. Lancet (2019) 394:420-31. doi: 10.1016/S0140-6736(19)31266-8

43. Klemenak M, Dolinsek J, Langerholc T, Di Gioia D, Micetic-Turk D. Administration of Bifidobacterium breve Decreases the Production of TNFalpha in Children with Celiac Disease. Dig Dis Sci (2015) 60:3386-92. doi: 10.1007/s10620-015-3769-7

44. Olivares M, Castillejo G, Varea V, Sanz Y. Double-blind, randomised, placebo-controlled intervention trial to evaluate the effects of Bifidobacterium longum CECT 7347 in children with newly diagnosed coeliac disease. Br J Nutr (2014) 112:30-40. doi: 10.1017/S0007114514000609

45. Pinto-Sanchez MI, Smecuol EC, Temprano MP, Sugai E, Gonzalez A, Moreno ML, et al. Bifidobacterium infantis NLS Super Strain Reduces the Expression of alpha-Defensin-5, a Marker of Innate Immunity, in the Mucosa of Active Celiac Disease Patients. J Clin Gastroenterol (2017) 51:814-7. doi: 10.1097/ MCG.0000000000000687

46. Smecuol E, Hwang HJ, Sugai E, Corso L, Chernavsky AC, Bellavite FP, et al. Exploratory, randomized, double-blind, placebo-controlled study on the effects of Bifidobacterium infantis natren life start strain super strain in active celiac disease. J Clin Gastroenterol (2013) 47:139-47. doi: 10.1097/ MCG.0b013e31827759ac

47. Francavilla R, Piccolo M, Francavilla A, Polimeno L, Semeraro F, Cristofori F, et al. Clinical and Microbiological Effect of a Multispecies Probiotic Supplementation in Celiac Patients With Persistent IBS-type Symptoms: A Randomized. Double-Blind Placebo-controlled Multicenter Trial J Clin Gastroenterol (2019) 53:e117-25. doi: 10.1097/MCG.0000000000001023

48. Hakansson A, Andren Aronsson C, Brundin C, Oscarsson E, Molin G, Agardh D. Effects of Lactobacillus plantarum and Lactobacillus paracasei on the Peripheral Immune Response in Children with Celiac Disease Autoimmunity: A Randomized, Double-Blind, Placebo-Controlled Clinical Trial. Nutrients (2019) 11:1-12. doi: 10.3390/nu11081925

49. Harnett J, Myers SP, Rolfe M. Probiotics and the Microbiome in Celiac Disease: A Randomised Controlled Trial. Evid Based Complement Alternat Med (2016) 2016:9048574. doi: 10.1155/2016/9048574

50. Jeon SG, Kayama H, Ueda Y, Takahashi T, Asahara T, Tsuji H, et al. Probiotic Bifidobacterium breve induces IL-10-producing Tr1 cells in the colon. PloS Pathog (2012) 8:e1002714. doi: 10.1371/journal.ppat.1002714

51. Zheng B, van Bergenhenegouwen J, Overbeek S, van de Kant HJ, Garssen J, Folkerts $\mathrm{G}$, et al. Bifidobacterium breve attenuates murine dextran sodium sulfate-induced colitis and increases regulatory $\mathrm{T}$ cell responses. PloS One (2014) 9:e95441. doi: 10.1371/journal.pone.0095441

52. Laparra JM, Olivares M, Gallina O, Sanz Y. Bifidobacterium longum CECT 7347 modulates immune responses in a gliadin-induced enteropathy animal model. PloS One (2012) 7:e30744. doi: 10.1371/journal.pone.0030744

53. O’Mahony C, Scully P, O'Mahony D, Murphy S, O’Brien F, Lyons A, et al. Commensal-induced regulatory $\mathrm{T}$ cells mediate protection against pathogenstimulated NF-kappaB activation. PloS Pathog (2008) 4:e1000112. doi: 10.1371/journal.ppat.1000112 
54. van der Kleij H, O’Mahony C, Shanahan F, O’Mahony L, Bienenstock J. Protective effects of Lactobacillus rhamnosus [corrected. and Bifidobacterium infantis in murine models for colitis do not involve the vagus nerve. Am J Physiol Regul Integr Comp Physiol (2008) 295:R1131-7. doi: 10.1152/ ajpregu.90434.2008

55. Tanabe S, Kinuta Y, Saito Y. Bifidobacterium infantis suppresses proinflammatory interleukin-17 production in murine splenocytes and dextran sodium sulfate-induced intestinal inflammation. Int $\mathrm{J} \mathrm{Mol} \mathrm{Med}$ (2008) 22:181-5. doi: 0.3892/ijmm_00000006

56. Stanisavljevic S, Dinic M, Jevtic B, Dedovic N, Momcilovic M, Dokic J, et al. Gut Microbiota Confers Resistance of Albino Oxford Rats to the Induction of Experimental Autoimmune Encephalomyelitis. Front Immunol (2018) 9:942. doi: 10.3389/fimmu.2018.00942

57. Kouchaki E, Tamtaji OR, Salami M, Bahmani F, Daneshvar Kakhaki R, Akbari $\mathrm{E}$, et al. Clinical and metabolic response to probiotic supplementation in patients with multiple sclerosis: A randomized, double-blind, placebocontrolled trial. Clin Nutr (2017) 36:1245-9. doi: 10.1016/j.clnu.2016.08.015

58. Tankou SK, Regev K, Healy BC, Cox LM, Tjon E, Kivisakk P, et al. Investigation of probiotics in multiple sclerosis. Mult Scler (2018) 24:58-63. doi: $10.1177 / 1352458517737390$

59. Vaghef-Mehrabany E, Alipour B, Homayouni-Rad A, Sharif SK, AsghariJafarabadi M, Zavvari S. Probiotic supplementation improves inflammatory status in patients with rheumatoid arthritis. Nutrition (2014) 30:430-5. doi: 10.1016/j.nut.2013.09.007

60. So JS, Kwon HK, Lee CG, Yi HJ, Park JA, Lim SY, et al. Lactobacillus casei suppresses experimental arthritis by down-regulating $\mathrm{T}$ helper 1 effector functions. Mol Immunol (2008) 45:2690-9. doi: 10.1016/j.molimm.2007.12.010

61. Kato I, Endo-Tanaka K, Yokokura T. Suppressive effects of the oral administration of Lactobacillus casei on type II collagen-induced arthritis in DBA/1 mice. Life Sci (1998) 63:635-44. doi: 10.1016/S0024-3205(98)00315-4

62. Amdekar S, Singh V, Singh R, Sharma P, Keshav P, Kumar A. Lactobacillus casei reduces the inflammatory joint damage associated with collagen-induced arthritis (CIA) by reducing the pro-inflammatory cytokines: Lactobacillus casei: COX-2 inhibitor. J Clin Immunol (2011) 31:147-54. doi: 10.1007/ s10875-010-9457-7

63. Zamani B, Golkar HR, Farshbaf S, Emadi-Baygi M, Tajabadi-Ebrahimi M, Jafari $\mathrm{P}$, et al. Clinical and metabolic response to probiotic supplementation in patients with rheumatoid arthritis: a randomized, double-blind, placebocontrolled trial. Int J Rheum Dis (2016) 19:869-79. doi: 10.1111/1756$185 X .12888$

64. Pineda Mde L, Thompson SF, Summers K, de Leon F, Pope J, Reid G. A randomized, double-blinded, placebo-controlled pilot study of probiotics in active rheumatoid arthritis. Med Sci Monit (2011) 17:CR347-54. doi: 10.12659/MSM.881808

65. Wang C, Zaheer M, Bian F, Quach D, Swennes AG, Britton RA, et al. SjogrenLike Lacrimal Keratoconjunctivitis in Germ-Free Mice. Int J Mol Sci (2018) 19:45-56. doi: 10.3390/ijms19020565

66. Zaheer M, Wang C, Bian F, Yu Z, Hernandez H, de Souza RG, et al. Protective role of commensal bacteria in Sjogren Syndrome. J Autoimmun (2018) 93:4556. doi: 10.1016/j.jaut.2018.06.004

67. Kamal Y, Kandil M, Eissa M, Yousef R, Elsaadany B. Probiotics as a prophylaxis to prevent oral candidiasis in patients with Sjogren's syndrome: a double-blinded, placebo-controlled, randomized trial. Rheumatol Int (2020) 40:873-9. doi: 10.1007/s00296-020-04558-9

68. Choi SH, Oh JW, Ryu JS, Kim HM, Im SH, Kim KP, et al. IRT5 Probiotics Changes Immune Modulatory Protein Expression in the Extraorbital Lacrimal Glands of an Autoimmune Dry Eye Mouse Model. Invest Ophthalmol Vis Sci (2020) 61:42. doi: 10.1167/iovs.61.3.42

69. Zare Javid A, Aminzadeh M, Haghighi-Zadeh MH, Jamalvandi M. The Effects of Synbiotic Supplementation on Glycemic Status, Lipid Profile, and Biomarkers of Oxidative Stress in Type 1 Diabetic Patients. A PlaceboControlled. Double-Blind Randomized Clin Trial Diabetes Metab Syndr Obes (2020) 13:607-17. doi: 10.2147/DMSO.S238867

70. Solanki HK, Shah DA, Maheriya PM, Patel CA. Evaluation of antiinflammatory activity of probiotic on carrageenan-induced paw edema in Wistar rats. Int J Biol Macromol (2015) 72:1277-82. doi: 10.1016/ j.ijbiomac.2014.09.059
71. Teixeira LD, Kling DN, Lorca GL, Gonzalez CF. Lactobacillus johnsonii N6.2 diminishes caspase-1 maturation in the gastrointestinal system of diabetes prone rats. Benef Microbes (2018) 9:527-39. doi: 10.3920/BM2017.0120

72. Valladares R, Sankar D, Li N, Williams E, Lai KK, Abdelgeliel AS, et al. Lactobacillus johnsonii N6.2 mitigates the development of type 1 diabetes in BB-DP rats. PloS One (2010) 5:e10507. doi: 10.1371/journal.pone.0010507

73. Lau K, Benitez P, Ardissone A, Wilson TD, Collins EL, Lorca G, et al. 3rd, Inhibition of type 1 diabetes correlated to a Lactobacillus johnsonii N6.2mediated Th17 bias. J Immunol (2011) 186:3538-46. doi: 10.4049/ jimmunol.1001864

74. Valladares R, Bojilova L, Potts AH, Cameron E, Gardner C, Lorca G, et al. Lactobacillus johnsonii inhibits indoleamine 2,3-dioxygenase and alters tryptophan metabolite levels in BioBreeding rats. FASEB J (2013) 27:171120. doi: 10.1096/fj.12-223339

75. Kingma SD, Li N, Sun F, Valladares RB, Neu J, Lorca GL. Lactobacillus johnsonii N6.2 stimulates the innate immune response through Toll-like receptor 9 in Caco- 2 cells and increases intestinal crypt Paneth cell number in biobreeding diabetes-prone rats. J Nutr (2011) 141:1023-8. doi: 10.3945/ jn.110.135517

76. Takiishi T, Korf H, Van Belle TL, Robert S, Grieco FA, Caluwaerts S, et al. Reversal autoimmune Diabetes by Restor antigen-specific tolerance using genetically modified Lactococcus lactis mice. J Clin Invest (2012) 122:1717-25. doi: 10.1172/JCI60530

77. Groele L, Szajewska H, Szypowska A. Effects of Lactobacillus rhamnosus GG and Bifidobacterium lactis Bb12 on beta-cell function in children with newly diagnosed type 1 diabetes: protocol of a randomised controlled trial. BMJ Open (2017) 7:e017178. doi: 10.1136/bmjopen-2017-017178

78. Marietta EV, Murray JA, Luckey DH, Jeraldo PR, Lamba A, Patel R, et al. Suppression of Inflammatory Arthritis by Human Gut-Derived Prevotella histicola in Humanized Mice. Arthritis Rheumatol (2016) 68:2878-88. doi: 10.1002/art.39785

79. Mangalam A, Shahi SK, Luckey D, Karau M, Marietta E, Luo NL, et al. Human Gut-Derived Commensal Bacteria Suppress CNS Inflammatory and Demyelinating Disease. Cell Rep (2017) 20:1269-77. doi: 10.1016/ j.celrep.2017.07.031

80. Jia L, Shan K, Pan LL, Feng N, Lv Z, Sun Y, et al. Clostridium butyricum CGMCC0313.1 Protects against Autoimmune Diabetes by Modulating Intestinal Immune Homeostasis and Inducing Pancreatic Regulatory $\mathrm{T}$ Cells. Front Immunol (2017) 8:1345. doi: 10.3389/fimmu.2017.01345

81. Hanninen A, Toivonen R, Poysti S, Belzer C, Plovier H, Ouwerkerk JP, et al. Akkermansia muciniphila induces gut microbiota remodelling and controls islet autoimmunity in NOD mice. Gut (2018) 67:1445-53. doi: 10.1136/gutjnl2017-314508

82. Bodkhe R, Marietta EV, Balakrishnan B, Luckey DH, Horwath IE, Shouche YS, et al. Human gut-derived commensal suppresses generation of T-cell response to gliadin in humanized mice by modulating gut microbiota. Anaerobe (2020) 102237. doi: 10.1016/j.anaerobe.2020.102237

83. McCarville JL, Dong J, Caminero A, Bermudez-Brito M, Jury J, Murray JA, et al. A Commensal Bifidobacterium longum Strain Prevents Gluten-Related Immunopathology in Mice through Expression of a Serine Protease Inhibitor. Appl Environ Microbiol (2017) 83:1-13. doi: 10.1128/AEM.01323-17

84. Olivares M, Laparra M, Sanz Y. Oral administration of Bifidobacterium longum CECT 7347 modulates jejunal proteome in an in vivo gliadininduced enteropathy animal model. J Proteomics (2012) 77:310-20. doi: 10.1016/j.jprot.2012.09.005

85. Orlando A, Linsalata M, Bianco G, Notarnicola M, D'Attoma B, Scavo MP, et al. Lactobacillus rhamnosus GG Protects the Epithelial Barrier of Wistar Rats from the Pepsin-Trypsin-Digested Gliadin (PTG)-Induced Enteropathy. Nutrients (2018) 10:1-16. doi: 10.3390/nu10111698

86. D’Arienzo R, Stefanile R, Maurano F, Mazzarella G, Ricca E, Troncone R, et al. Immunomodulatory effects of Lactobacillus casei administration in a mouse model of gliadin-sensitive enteropathy. Scand J Immunol (2011) 74:335-41. doi: 10.1111/j.1365-3083.2011.02582.x

87. He B, Hoang TK, Tian X, Taylor CM, Blanchard E, Luo M, et al. Lactobacillus reuteri Reduces the Severity of Experimental Autoimmune Encephalomyelitis in Mice by Modulating Gut Microbiota. Front Immunol (2019) 10:385. doi: 10.3389/fimmu.2019.00385 
88. Maassen CB, Claassen E. Strain-dependent effects of probiotic lactobacilli on EAE autoimmunity. Vaccine (2008) 26:2056-7. doi: 10.1016/j.vaccine.2008.02.035

89. an H, Guo R, Ju Y, Wang Q, Zhu J, Xie Y, et al. A single bacterium restores the microbiome dysbiosis to protect bones from destruction in a rat model of rheumatoid arthritis. Microbiome (2019) 7:107. doi: 10.1186/s40168-019-0719-1

90. Cardoso RS, Messora MR, Silva PHF, Oliveira LF, Leite-Panissi C, Salvador S, et al. Effects of Bifidobacterium animalis subsp. lactis HN019 on ligatureinduced periodontitis in rats with experimental rheumatoid arthritis. Benef Microbes (2020) 11:33-46. doi: 10.3920/BM2019.0038

91. Esvaran M, Conway PL. Lactobacillus fermentum PC1 has the Capacity to Attenuate Joint Inflammation in Collagen-Induced Arthritis in DBA/1 Mice. Nutrients (2019) 11:1-9. doi: 10.3390/nu11040785

92. Gohil P, Patel V, Deshpande S, Chorawala M, Shah G. Anti-arthritic activity of cell wall content of Lactobacillus plantarum in freund's adjuvant-induced arthritic rats: involvement of cellular inflammatory mediators and other biomarkers. Inflammopharmacology (2018) 26:171-81. doi: 10.1007/s10787-017-0370-z

93. Nowak B, Ciszek-Lenda M, Srottek M, Gamian A, Kontny E, Gorska-Fraczek S, et al. Lactobacillus rhamnosus exopolysaccharide ameliorates arthritis induced by the systemic injection of collagen and lipopolysaccharide in DBA/1 mice. Arch Immunol Ther Exp (Warsz) (2012) 60:211-20. doi: 10.1007/s00005-0120170-5

94. Nowak B, Srottek M, Ciszek-Lenda M, Skalkowska A, Gamian A, Gorska S, et al. Exopolysaccharide from Lactobacillus rhamnosus KL37 Inhibits T Celldependent Immune Response in Mice. Arch Immunol Ther Exp (Warsz) (2020) 68:17. doi: 10.1007/s00005-020-00581-7
95. Abdelazez A, Abdelmotaal H, Evivie SE, Melak S, Jia FF, Khoso MH, et al. Screening Potential Probiotic Characteristics of Lactobacillus brevis Strains In Vitro and Intervention Effect on Type I Diabetes In Vivo. BioMed Res Int (2018) 2018:7356173. doi: 10.1155/2018/7356173

96. Huibregtse IL, Marietta EV, Rashtak S, Koning F, Rottiers P, David CS, et al. Induction of antigen-specific tolerance by oral administration of Lactococcus lactis delivered immunodominant DQ8-restricted gliadin peptide in sensitized nonobese diabetic Abo Dq8 transgenic mice. J Immunol (2009) 183:2390-6. doi: 10.4049/jimmunol.0802891

97. Maassen CB, Laman JD, van Holten-Neelen C, Hoogteijling L, Groenewegen L, Visser L, et al. Reduced experimental autoimmune encephalomyelitis after intranasal and oral administration of recombinant lactobacilli expressing myelin antigens. Vaccine (2003) 21:4685-93. doi: 10.1016/S0264-410X(03) 00522-X

Conflict of Interest: The authors are all co-inventors on US patent \#10,555,975 B2, entitled: Prevotella Histicola Preparations and the Treatment of Autoimmune Conditions. VT received funding from Evelo Biosciences, Inc.

Copyright (C) 2020 Marietta, Mangalam, Taneja and Murray. This is an open-access article distributed under the terms of the Creative Commons Attribution License (CC BY). The use, distribution or reproduction in other forums is permitted, provided the original author(s) and the copyright owner(s) are credited and that the original publication in this journal is cited, in accordance with accepted academic practice. No use, distribution or reproduction is permitted which does not comply with these terms. 